\title{
Generation of Low-Attenuation Lamb Wave Modes in Three-Layer Adhesive Joints
}

\author{
Pezhman Taghipour Birgani \\ Department of Mechanical Engineering, Ahvaz Branch, Islamic Azad University, Ahvaz, Iran
}

\begin{abstract}
Sina Sodagar
Department of Technical Inspection Engineering, Abadan Faculty of Petroleum Engineering, Petroleum University of Technology, Iran
\end{abstract}

\author{
Mohammad Shishesaz \\ Department of Mechanical Engineering, Shahid Chamran University, Ahvaz, Iran
}

\begin{abstract}
(Received 22 October 2014; accepted 29 March 2016)
In this paper, the attenuation of lamb waves in three-layer adhesive joints, including two elastic plates bonded together by a viscoelastic adhesive layer, is investigated using the global matrix method and then a suitable incidence angle is calculated to generate a low-attenuation lamb wave using an angle beam transducer. The theoretical boundary value problem in adhesive joints with a perfect bond and traction-free boundary conditions on their outer surfaces is solved to find a combination of frequencies and modes with lowest attenuation. A characteristic equation is derived by applying continuity and boundary conditions in adhesive joints using the global matrix method. Phase velocity and attenuation dispersion curves are obtained with numerical solution of this equation by a computer code for a three-layer joint, including an aluminum repair patch bonded to the aircraft aluminum skin by a layer of viscoelastic epoxy adhesive. To validate the numerical solution results, wave structure curves are plotted for a special mode in two different frequencies in the adhesive joint. Also, the transducer incidence angle is calculated in terms of frequency to generate lamb wave modes with low attenuation level using the theoretical method.
\end{abstract}

\section{INTRODUCTION}

The applications of adhesive joints in plates and pipes to increase life, improve stiffness, and protect against corrosion and electrical insulation have resulted the widespread use of viscoelastic polymer material like epoxy with plates and pipes as adhesive and coating. An example of these joints' application is a three-layer adhesive joint, including an aluminum patch bonded to a surface, such as aircraft aluminum skin, by a viscoelastic epoxy adhesive layer. Repair patches are used to extend the life of the aircraft. Ultrasonic guided waves are used to inspect these adhesive joints. Lamb waves have applications in non-destructive inspection of elastic-viscoelastic multi-layer joints and plates. Some modes of these waves have frequencies with minimum attenuation and are recognizable in inspection by a transducer, and they can also detect the defects in the structures. Low-attenuation lamb waves can be produced in multi-layer structures using angle beam transducers for inspection purposes.

Guided waves propagation in multi-layer structures has been investigated using various methods. The transfer matrix method is applied to model guided waves in multilayered anisotropic and damping media, the use of this method in high frequencies is accompanied with numerical instability. ${ }^{1}$ Delta operator technique is applied to improve the stability of transfer matrix method in multilayered anisotropic damping plates. ${ }^{2}$ The matrix methods have been presented for modeling the propagation of ultrasonic waves in multilayered media. ${ }^{3}$ Both global the matrix method and transfer matrix method are used in these studies. These techniques can be used to obtain attenuation and phase velocity dispersion curves in viscoelastic materials. The propagation of guided waves in multilayered adhesive structures has been investigated using analytical, experimental, and transient FEM simulation methods by taking into consideration the low stiffness and viscoelastic behavior of adhesive layer. ${ }^{4}$

The propagation of lamb and shear horizontal (SH) waves in elastic plates coated with viscoelastic materials has been investigated by Superposition Partial Bulk Waves (SPBW) method. ${ }^{5,6}$ Material damping causes an excessive reduction of an applied signal in an ultrasonic test. The propagation of guided waves in elastic hollow cylinders with viscoelastic coating has been investigated using experimental and analytical methods. ${ }^{7}$ Wave equation in elastic hollow cylinders was solved using theoretical boundary value problem and the best modes were specified. The global matrix method has been used to obtain the roots of the characteristic equation. Wave propagation in linear viscoelastic laminates has been investigated using spectral finite element method or semi-analytical finite element method (SAFE), the damping loss factor was estimated for waves in low frequencies, and the stiffness matrix was assumed to be real. ${ }^{8}$ The damping loss factor has been obtained using the SAFE method and taking into consideration the complex stiffness matrix. ${ }^{9}$ To model ultrasonic wave propagation in different waveguides, the SAFE method is used. ${ }^{10}$ The titanium repair patches bonded to the aircraft aluminum skin have been inspected using ultrasonic guided waves, wave structures were plotted using a theoretical method and selected the mode shape with maximum in-plane displacement for inspection, although the effect of material damping did not take into consideration. ${ }^{11}$

In the present study, the propagation of lamb waves in 


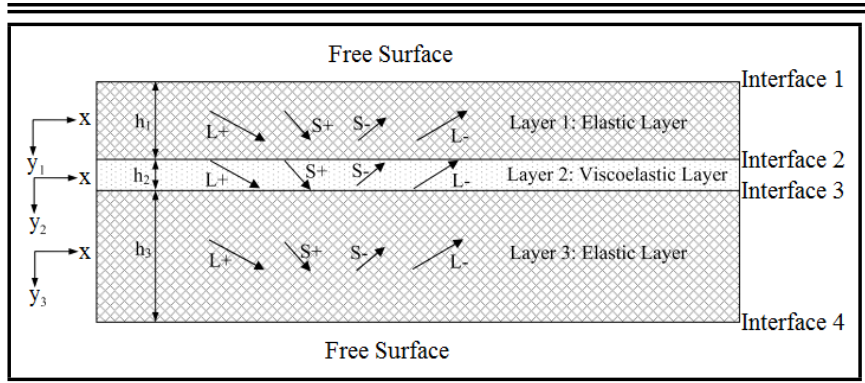

Figure 1. The propagation of the lamb wave in an elastic-viscoelastic threelayer adhesive joint.

elastic-viscoelastic three-layer joints, including two elastic plates bonded together with a layer of viscoelastic adhesive, is investigated using the global matrix method and considering the viscoelastic layer damping effect. Then, the suitable incidence angle is theoretically calculated to generate lamb wave mode with low attenuation using an angle beam transducer. Adhesive damping causes an excessive reduction in the sending signal amplitude in ultrasonic tests. Hence, modes and frequencies with minimum attenuation should be specified because these waves travel the maximum possible distance in joints and can detect the different defects namely interfacial defects.

\section{LAMB WAVES PROPAGATION IN THREE- LAYER ADHESIVE JOINTS}

Lamb waves are propagated in thin plate-like mediums in which planar dimensions are far greater than the thickness of the plate and wavelength of the same order with plate thickness. ${ }^{12}$ Free upper and lower surfaces of plate lead movement of these waves. Lamb waves have infinite modes and their propagation properties depend on wave entry angle, frequency, and structure geometry. Figure 1 shows lamb wave propagation in an adhesive joint, which is comprised of three layers. The first and the third layers, which are elastic and isotropic, are bonded together by the second layer, which is an isotropic viscoelastic layer. The layers are perfectly bonded together and the free surfaces at the top and the bottom of the three layers are traction-free. Layer thickness is shown using $h_{1}$, $h_{2}$, and $h_{3}$. A local Cartesian coordinate system is used to investigate the propagation of lamb waves in the three layers. Because of the propagation of the lamb waves in the threelayer joint, the problem is investigated as plain strain and the wave propagation is considered to be harmonic. In each layer, lamb waves are comprised of shear and longitudinal waves superposition. ${ }^{13}$ Symbols $L+$ and $L-$ show the propagation of longitudinal waves downwards and upwards the plate, and $S+$ and $S$ - show the propagation of shear waves downwards and upwards the plate, respectively.

Assuming that the wave propagation in three-layer adhesive joints in terms of time is harmonic, the stress-strain equations of the viscoelastic layer are similar to those in the elastic layer, except that material properties of the viscoelastic layer are complex numbers and a function of frequency. ${ }^{14}$ This dependency between elastic and viscoelastic materials in a harmonic state is called Alfrey's Correspondence Principle. Also, Navier's equation of motion in the viscoelastic layer is similar to the elastic layer and is expressed by Eq. (1).

$$
\mu \nabla^{2} \mathbf{u}+(\lambda+\mu) \nabla(\nabla \mathbf{u})=\rho \frac{\partial^{2} \mathbf{u}}{\partial t^{2}}
$$

In Eq. (1), $\lambda, \mu$, and $\rho$ are Lame constants and density, respectively. Lame constants in viscoelastic material are complex numbers and a function of frequency that are measured using experimental methods such as ultrasonic tests. ${ }^{7}$ In Eq. (1), the displacement field, $\mathbf{u}$, can be decomposed as a combination of the gradient of a scalar potential field, $\Phi$, and the curl of a vector potential field, $\mathbf{H}$ (Helmholtz decomposition). ${ }^{13}$

$$
\mathbf{u}=\nabla \Phi+\nabla \times \mathbf{H}, \quad \nabla \mathbf{H}=0 .
$$

Substituting Eq. (2) in Eq. (1), scalar and vector equations are obtained respectively:

$$
\begin{aligned}
\nabla^{2} \Phi & =\frac{1}{C_{1}^{2}} \frac{\partial^{2} \Phi}{\partial t^{2}}, & C_{1} & =\sqrt{\frac{\lambda+2 \mu}{\rho} ;} \\
\nabla^{2} \mathbf{H} & =\frac{1}{C_{2}^{2}} \frac{\partial^{2} \mathbf{H}}{\partial t^{2}}, & C_{2} & =\sqrt{\frac{\mu}{\rho}} ;
\end{aligned}
$$

Equation (3) shows the propagation of longitudinal wave, Eq. (4) shows the propagation of the transverse wave in structures, and $C_{1}$ and $C_{2}$ quantities are the longitudinal and shear wave velocities in medium, respectively. Since Lame constants in viscoelastic material are complex numbers and a function of frequency, wave velocities are also complex numbers and a function of frequency.

Using the Cartesian coordinate system, the potential vector, $\mathbf{H}$, can be defined as the Eq. (5):

$$
\mathbf{H}=H_{x} \mathbf{e}_{x}+H_{y} \mathbf{e}_{y}+H_{z} \mathbf{e}_{z}
$$

Since this problem is assumed as a plain strain, then the equation $u_{z}=\partial / \partial z=0$ should be satisfied. This happens when the $H_{x}$ and $H_{y}$ components equal zero and only $H_{z}$ remains. The scalar potential function, $\Phi$, should also be a function of $x$ and $y$.

The solutions of Eqs. (3) and (4) for a harmonic wave propagate along the positive $x$ direction and are assumed to be Eqs. (6) and (7):

$$
\begin{aligned}
\Phi & =f(y) e^{i(k x-\omega t)} \\
H_{z} & =h_{z}(y) e^{i(k x-\omega t)} .
\end{aligned}
$$

In Eqs. (6) and (7), $k$ and $\omega$ are the wave number and angular frequency, respectively.

Substituting Eqs. (6) and (7) in Eqs. (3) and (4) and taking into consideration that the two components of the vector potential function are equal to zero, and after solving the differential equations, the solutions are obtained as:

$$
\begin{gathered}
\Phi=\left\{A_{(L+)} e^{i \alpha y}+A_{(L-)} e^{-i \alpha y}\right\} e^{i(k x-\omega t)} \\
\alpha^{2}=\left(\frac{\omega^{2}}{C_{1}^{2}}-k^{2}\right) \\
H_{z}=\left\{A_{(S+)} e^{i \beta y}+A_{(S-)} e^{-i \beta y}\right\} e^{i(k x-\omega t)} \\
\beta^{2}=\left(\frac{\omega^{2}}{C_{2}^{2}}-k^{2}\right)
\end{gathered}
$$

The solutions of Eqs. (8) and (9) are known as the partial waves solution. The four terms obtained from Eqs. (8) and (9) show the longitudinal waves propagation $L$, and the transverse waves propagation $S$, upwards and downwards the layer. Constant values show the amplitude of propagated waves. For 
instance, $A_{(L+)}$ shows that the longitudinal wave amplitude propagates towards the bottom of the layer.

Substituting vector and scalar potential functions from Eqs. (8) and (9) in Eq. (2), the displacement field in adhesive joint is obtained in terms of unknown constants of the shear and longitudinal wave amplitudes:

$$
\begin{aligned}
& u_{x}=i\left\{k\left(A_{(L+)} e^{i \alpha y}+A_{(L-)} e^{-i \alpha y}\right)+\right.\left.\beta\left(A_{(S+)} e^{i \beta y}-A_{(S-)} e^{-i \beta y}\right)\right\} e^{i(k x-\omega t)} ; \\
& u_{y}=i\left\{\alpha\left(A_{(L+)} e^{i \alpha y}-A_{(L-)} e^{-i \alpha y}\right)-\right. \\
&\left.\quad k\left(A_{(S+)} e^{i \beta y}+A_{\left(S_{-}\right)} e^{-i \beta y}\right)\right\} e^{i(k x-\omega t)} ; \\
& u_{z}=0 .
\end{aligned}
$$

Equations (10) and (11) can also be expressed as Eqs. (13) and (14):

$$
\begin{aligned}
& u_{x}=U_{x} e^{i(k x-\omega t)} \\
& u_{y}=U_{y} e^{i(k x-\omega t)} .
\end{aligned}
$$

In Eqs. (13) and (14), $U_{x}$ and $U_{y}$ are unattenuated displacement amplitudes.

Using Hooke and strain-displacement relations, stresses in the adhesive joint can be obtained in terms of the unknown constants of shear and longitudinal wave amplitudes:

$$
\begin{aligned}
& \sigma_{x x}= \mu\left\{\left(2 \alpha^{2}-k^{2}-\beta^{2}\right)\left(A_{(L+)} e^{i \alpha y}+A_{(L-)} e^{-i \alpha y}\right)-\right. \\
&\left.2 k \beta\left(A_{(S+)} e^{i \beta y}-A_{(S-)} e^{-i \beta y}\right)\right\} e^{i(k x-\omega t)} ; \quad(15) \\
& \sigma_{y y}= \mu\left\{\left(k^{2}-\beta^{2}\right)\left(A_{(L+)} e^{i \alpha y}+A_{(L-)} e^{-i \alpha y}\right)+\right. \\
&\left.2 k \beta\left(A_{(S+)} e^{i \beta y}-A_{(S-)} e^{-i \beta y}\right)\right\} e^{i(k x-\omega t)} ; \quad(16) \\
& \sigma_{z z}=-\lambda\left\{\left(\alpha^{2}+k^{2}\right)\left(A_{(L+)} e^{i \alpha y}+A_{(L-)} e^{-i \alpha y}\right)\right\} e^{i(k x-\omega t)} ; \\
& \sigma_{x y}=-\mu\left\{2 k \alpha\left(A_{(L+)} e^{i \alpha y}-A_{(L-)} e^{-i \alpha y}\right)+\right. \\
&\left.\left(\beta^{2}-k^{2}\right)\left(A_{(S+)} e^{i \beta y}+A_{(S-)} e^{-i \beta y}\right)\right\} e^{i(k x-\omega t)} ; \quad(18) \\
& \sigma_{x z}=0 ; \quad \sigma_{y z}=0 .
\end{aligned}
$$

In order to obtain lamb waves dispersion curves for the elastic-viscoelastic three-layer adhesive joint, continuity and boundary conditions need to be applied.

\section{FORMULATION OF CONTINUITY AND BOUNDARY CONDITIONS}

The global matrix method is suitable for the formulation of problems concerning multi-layers. Continuity and boundary conditions are needed for this formulation. Using this method, continuity and boundary conditions can be shown as matrices and vectors. This method can simultaneously consider effects of material damping and wave leakage to the environment. In this method a global matrix is used to describe all the continuity and boundary conditions, and when it comes to numerical stability, it is better than other matrix methods. ${ }^{3}$

Figure 2 shows the boundary conditions of a three-layer adhesive joint including stress and displacement continuity in

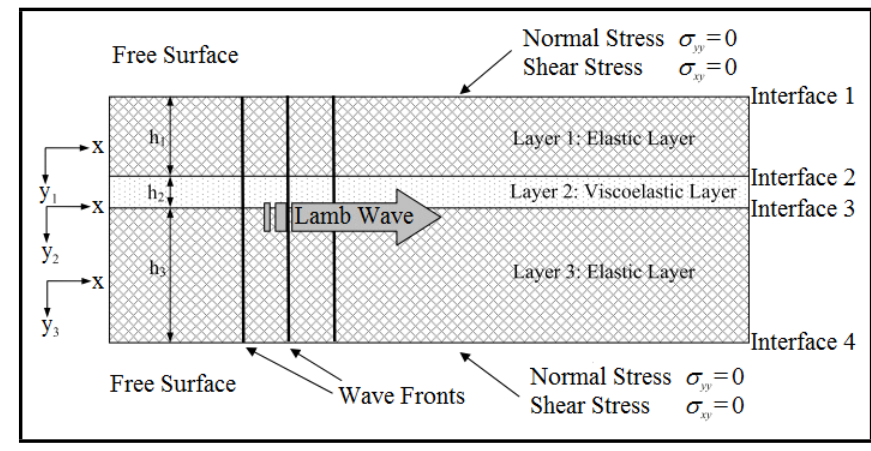

Figure 2. Continuity and boundary conditions in an elastic-viscoelastic threelayer adhesive joint.

layers interfaces and traction-free conditions in up and bottom surfaces of the elastic-viscoelastic three-layer adhesive joint.

The bond between the layers is perfect, and there's no shear and normal stress on the free-surfaces at the top and bottom of the three-layer adhesive joint. This condition is shown in Eq. (20). Continuity of interface conditions include continuity of the displacement, shear, and normal stresses components. As an example, continuity between the $m$ and $m+1$ layers are shown by vector Eq. (21).

$$
\left\{\begin{array}{l}
\sigma_{y y} \\
\sigma_{x y}
\end{array}\right\}_{\substack{\text { Free } \\
\text { surface }}}=\mathbf{0}
$$

$$
\left\{\begin{array}{c}
u_{x} \\
u_{y} \\
\sigma_{y y} \\
\sigma_{x y}
\end{array}\right\}_{\substack{\text { Layer }=m \\
\text { Interface }=m+1}}=\left\{\begin{array}{c}
u_{x} \\
u_{y} \\
\sigma_{y y} \\
\sigma_{x y}
\end{array}\right\}_{\substack{\text { Layer }=m+1 \\
\text { Interface }=m+1}} .
$$

Before applying continuity and boundary conditions, a vector relation for displacement and stress in each layer is necessary, which is obtained using Eqs. (10), (11), (16), and (18) and is shown by Eq. (22):

$$
\left\{\begin{array}{c}
u_{x} \\
u_{y} \\
\sigma_{y y} \\
\sigma_{x y}
\end{array}\right\}=\mathbf{D}\left\{\begin{array}{l}
A_{(L+)} \\
A_{(L-)} \\
A_{(S+)} \\
A_{(S-)}
\end{array}\right\} e^{i(k x-\omega t)}
$$

in which $\mathbf{D}$ is the layer matrix and is expressed as Eq. (23):

$$
\begin{aligned}
& \mathbf{D}= \\
& {\left[\begin{array}{cccc}
i k e^{i \alpha y} & i k e^{-i \alpha y} & i \beta e^{i \beta y} & -i \beta e^{-i \beta y} \\
i \alpha e^{i \alpha y} & -i \alpha e^{-i \alpha y} & -i k e^{i \beta y} & -i k e^{-i \beta y} \\
\mu\left(k^{2}-\beta^{2}\right) e^{i \alpha y} & \mu\left(k^{2}-\beta^{2}\right) e^{-i \alpha y} & 2 \mu k \beta e^{i \beta y} & -2 \mu k \beta e^{-i \beta y} \\
-2 \mu k \alpha e^{i \alpha y} & 2 \mu k \alpha e^{-i \alpha y} & \mu\left(k^{2}-\beta^{2}\right) e^{i \beta y} & \mu\left(k^{2}-\beta^{2}\right) e^{-i \beta y}
\end{array}\right] .}
\end{aligned}
$$

Before applying continuity and boundary conditions using Eq. (22), the layer matrix in the interfaces of each layer was calculated. This was achieved from Eq. (23) by substituting $y=-h / 2$ for layer top interface, and $y=h / 2$ for layer bottom interface. These two new layer matrices are shown by $\mathbf{D}_{t}$, $\mathbf{D}_{b}$, respectively, in which the subscripts $t$ and $b$ show the top and bottom interfaces of layer, respectively. Local coordinate system is used to derive these matrices, which are shown in Fig. 2, and therefore can be derived for all layers by substituting material properties and thickness.

Now, the three-layer joint continuity and boundary conditions are expressed in the form of a global matrix, which is 
shown in Eq. (24). The $\mathbf{A}_{m}, \mathbf{0}$ vectors in this matrix are shown by Eq. (25):

$$
\begin{gathered}
{\left[\begin{array}{ccc}
\left(\mathbf{D}_{1 t}\right)_{34} & \mathbf{0} & \mathbf{0} \\
\mathbf{D}_{1 b} & -\mathbf{D}_{2 t} & \mathbf{0} \\
\mathbf{0} & \mathbf{D}_{2 b} & -\mathbf{D}_{3 t} \\
\mathbf{0} & \mathbf{0} & \left(\mathbf{D}_{3 b}\right)_{34}
\end{array}\right]\left\{\begin{array}{l}
\mathbf{A}_{1} \\
\mathbf{A}_{2} \\
\mathbf{A}_{3}
\end{array}\right\}=\left\{\begin{array}{l}
\mathbf{0} \\
\mathbf{0} \\
\mathbf{0}
\end{array}\right\} ;} \\
\mathbf{A}_{m}=\left\{\begin{array}{l}
A_{(L+) m} \\
A_{(L-) m} \\
A_{(S+) m} \\
A_{(S-) m}
\end{array}\right\} ; \quad \mathbf{0}=\left\{\begin{array}{l}
0 \\
0 \\
0 \\
0
\end{array}\right\}
\end{gathered}
$$

In Eq. (24), the subscript 34 shows rows 3 and 4 of the layer matrix.

The global matrix method is a $4 n \times 4 n$ system of equations, in which $n$ is the number of layers and the global matrix for an elastic-viscoelastic three-layer is $12 \times 12$. In order for the nontrivial solution to exist, the determinant of global matrix should be zero. This is shown by Eq. (26), which is the characteristic or dispersion equation of lamb waves. With the aid of the roots of Eq. (26), attenuation and phase velocity dispersion curves are plotted in terms of frequency.

$$
\left|\begin{array}{ccc}
\left(\mathbf{D}_{1 t}\right)_{34} & \mathbf{0} & \mathbf{0} \\
\mathbf{D}_{1 b} & -\mathbf{D}_{2 t} & \mathbf{0} \\
\mathbf{0} & \mathbf{D}_{2 b} & -\mathbf{D}_{3 t} \\
\mathbf{0} & \mathbf{0} & \left(\mathbf{D}_{3 b}\right)_{34}
\end{array}\right|=0
$$

\section{THE NUMERICAL SOLUTION METHOD OF A CHARACTERISTIC EQUATION}

Characteristic equation roots in the three-layer adhesive joint are obtained using numerical solution method. In characteristic equation, frequency, $\omega$, is the independent variable, and wave number, $k$, is the dependent variable. The wave number in a desired frequency is obtained by solving this equation. To find characteristic equation roots, a computer code was written in MATLAB. These roots are shown by dispersion curves.

Finding complex roots of a characteristic equation concerning a three-layer adhesive joint of which at least one layer is viscoelastic is a difficult task. 4- If harmonic wave propagation is desired in linear viscoelastic material, then transverse and longitudinal velocities, and Lame constants of the viscoelastic layer, are complex and a function of frequency. 4- If harmonic wave propagation is desired in linear viscoelastic material, then transverse and longitudinal velocities, and Lame constants of the viscoelastic layer, are complex and a function of frequency. If harmonic wave propagation is desired in linear viscoelastic material, then transverse and longitudinal velocities, and Lame constants of the viscoelastic layer, are complex and are a function of frequency. The transverse and longitudinal velocities are calculated from Eqs. (27) and (28). ${ }^{14}$

$$
\begin{aligned}
& C_{1}(i \omega)=\frac{1}{\frac{1}{c_{1}(\omega)}+i \frac{\alpha_{1}(\omega)}{\omega}} ; \\
& C_{2}(i \omega)=\frac{1}{\frac{1}{c_{2}(\omega)}+i \frac{\alpha_{2}(\omega)}{\omega}} ;
\end{aligned}
$$

In Eqs. (27) and (28), $c_{1}$ and $c_{2}$ are bulk velocities of longitudinal and transverse waves and $\alpha_{1}$ and $\alpha_{2}$ are bulk attenuations of longitudinal and transverse waves of the viscoelastic layer. Bulk attenuation and velocity values for viscoelastic material

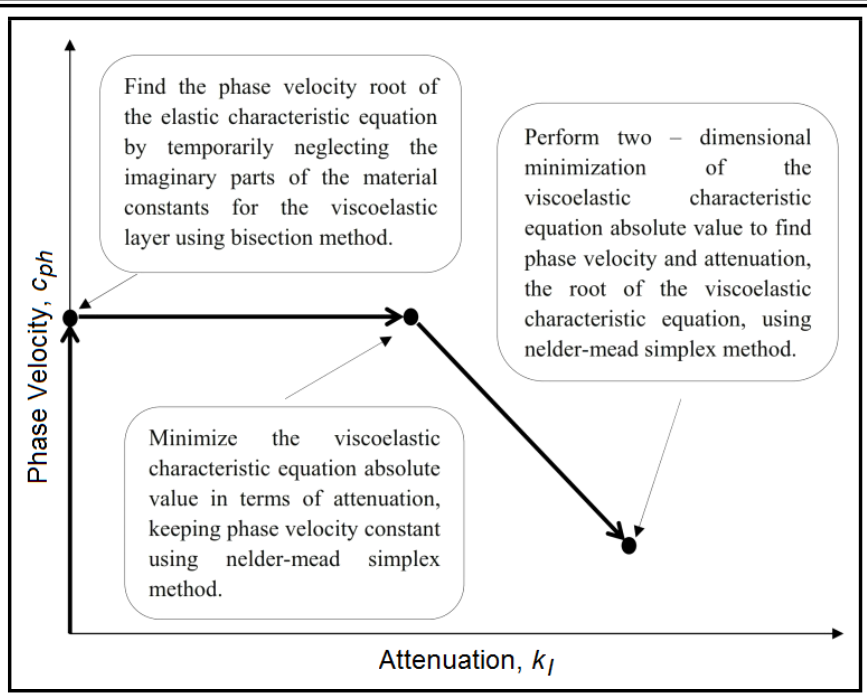

Figure 3. The process of minimization in order to find complex roots of the characteristic equation.

can be calculated in terms of frequency by using experimental tests such as the ultrasonic test. ${ }^{7}$

Before introducing a method for finding the attenuation and phase velocity numerical results, the wave number should be defined in terms of imaginary and real parts. Equation (29) shows the wave number as complex. ${ }^{15}$

$$
k=k_{R}+i k_{I}=\frac{\omega}{c_{p h}}+i k_{I} .
$$

Equation (29) enables us to solve the viscoelastic characteristic equation in terms of attenuation $k_{I}$, and phase velocity $c_{p h}$, instead of wave number $k$. In this case, the attenuation and phase velocity dispersion curves are obtained directly.

One solution method for finding the viscoelastic characteristic equation roots is taking into consideration the minimum of characteristic equation absolute value. In this case, the problem becomes three dimensional in which the characteristic equation absolute value is a function in terms of the attenuation and phase velocity. In this method, it is sought to find minimum value of this function. The main issue in this method is finding all the roots.

Figure 3 shows a minimization process of characteristic equation absolute value in order to find characteristic equation complex roots.

Using the process shown in Fig. 3, a computer code can be written to find characteristic equation roots. This process can be applied for all desired frequencies, and attenuation and phase velocity can be obtained in terms of frequency. The attenuation constant can be converted to attenuation in decibel per length unit by using Eq. (30). This conversion magnifies the attenuation values.

$$
\alpha_{0}\left(\mathrm{~dB} \mathrm{~m}^{-1}\right)=20 \log _{10}\left(e^{-1000 k_{I}}\right) .
$$

\section{LAMB WAVE MODE GENERATION}

Different methods exist to generate and receive ultrasonic guided waves. One method to generate a lamb wave is by using a longitudinal wave transducer on a plexiglass wedge, which is also called an angle beam transducer. Figure 4 shows the lamb wave generation method in an elastic-viscoelastic three-layer adhesive joint using an angle beam transducer. According to 


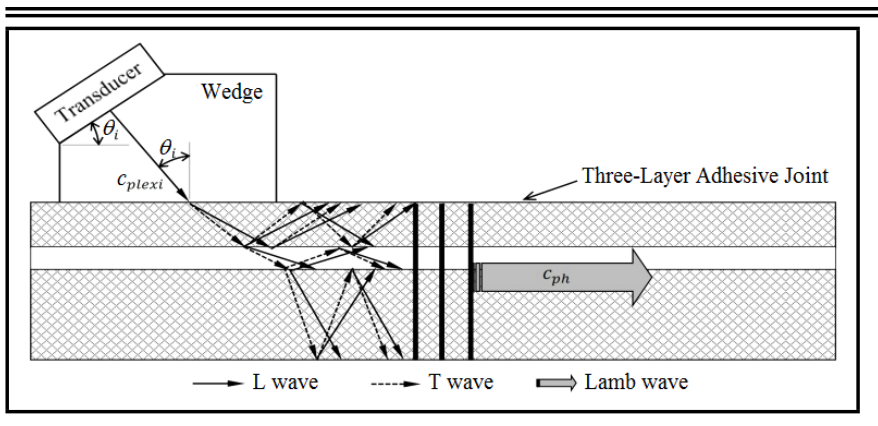

Figure 4. Lamb wave generation with an angle beam transducer.

Table 1. Geometric and acoustical properties of an elastic-viscoelastic threelayer adhesive joint. ${ }^{7}$

\begin{tabular}{|c|c|c|c|c|c|c|c|}
\hline Layer & Material & $\begin{array}{c}c_{1} \\
(\mathrm{~km} / \mathrm{s})\end{array}$ & $\begin{array}{c}\alpha_{1} / \omega \\
(\mathrm{s} / \mathrm{km})\end{array}$ & $\begin{array}{c}c_{2} \\
(\mathrm{~km} / \mathrm{s})\end{array}$ & $\begin{array}{c}\alpha_{2} / \omega \\
(\mathrm{s} / \mathrm{km})\end{array}$ & $\begin{array}{c}\rho \\
\left(\mathrm{g} / \mathrm{cm}^{3}\right)\end{array}$ & $\begin{array}{c}h \\
(\mathrm{~mm})\end{array}$ \\
\hline 1 & Aluminum & 6.35 & - & 3.13 & - & 2.7 & 1.6 \\
\hline 2 & $\begin{array}{c}\text { Mereco } \\
303 \text { Epoxy }\end{array}$ & 2.39 & 0.0070 & 0.99 & 0.0201 & 1.08 & 0.66 \\
\hline 3 & Aluminum & 6.35 & - & 3.13 & - & 2.7 & 3.175 \\
\hline
\end{tabular}

Snell's law, in this method, the transducer incidence angle depends on the wedge velocity and lamb wave phase velocity. Equation (31) shows Snell's law, in which $\theta_{i}$ is the plexiglass wedge angle, $c_{\text {plexi }}$ is the longitudinal wave velocity of the wedge, and $c_{p h}$ is the lamb wave phase velocity in adhesive joint.

$$
\theta_{i}=\sin ^{-1}\left(\frac{c_{p l e x i}}{c_{p h}}\right)
$$

In order to complete the theoretical modeling of the lamb wave generation, the attenuation and phase velocity in the joint are obtained first, then a combination of modes and frequencies that have low attenuation are selected, and finally the suitable incidence angle for generation is calculated using Snell's law and phase velocity of these modes. These angles are used in inspections.

\section{DISCUSSION OF RESULTS FOR A SPECIFIC APPLICATION}

Solving the characteristic equation by a computer code for a three-layer adhesive joint, including an aluminum repair patch bonded to the aircraft aluminum skin with a viscoelastic epoxy adhesive layer, the attenuation and phase velocity dispersion curves in high and low frequencies for this specific application are generated. Also, acceptable attenuation level is calculated for ultrasonic inspection using a single transducer of the adhesive joint with $200 \mathrm{~mm}$ length and suitable modes are selected. Geometric and acoustic properties of elastic-viscoelastic threelayer adhesive joint can be seen in Table 1. Aluminum and Mereco Epoxy 303 acoustic properties are picked up from. ${ }^{7}$

Wave structure for a mode in two different frequencies is plotted to validate numerical solution results. Finally, the transducer incidence angle is plotted in terms of frequency for different modes, and suitable wedge angles are selected to generate low-attenuation lamb wave modes in the adhesive joint with $200 \mathrm{~mm}$ length.

\subsection{Phase Velocity and Attenuation Dispersion Curves}

Figure 5 shows the phase velocity dispersion curve in terms of frequency for different modes in an elastic-viscoelastic three-layer adhesive joint, the properties of which are shown

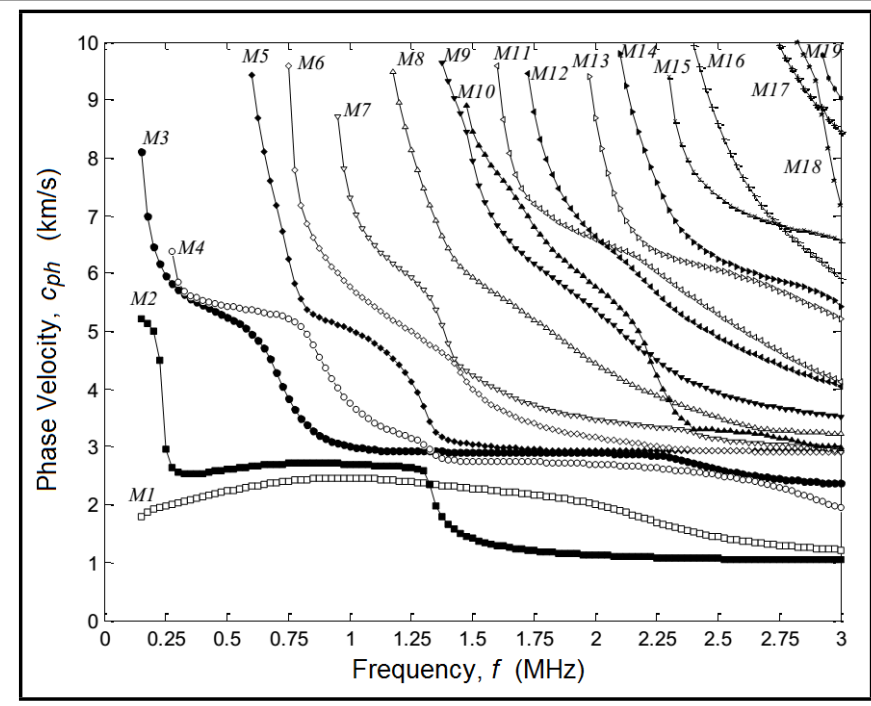

Figure 5. Phase velocity dispersion curves in terms of frequency in the threelayer adhesive joint: aluminum-epoxy-aluminum.

in Table 1. The lamb wave modes are identified with $M$ and numbers in Fig. 5. Investigating this curve, it can be seen that in the frequency intervals of $150 \mathrm{kHz}$ to $250 \mathrm{kHz}$ only three modes of $M 1, M 2$, and $M 3$ propagate, and other modes do not propagate in these low frequencies. $250 \mathrm{kHz}$ frequency is cutoff frequency of $M 4$ mode; because $M 4$ mode does not propagate in frequencies lower than this frequency. Also, in the frequency intervals of $275 \mathrm{kHz}$ to $575 \mathrm{kHz}$ only four modes of $M 1, M 2, M 3$, and $M 4$ propagate, and $575 \mathrm{kHz}$ is the cutoff frequency of $M 5$ mode.

Figure 6 shows the attenuation dispersion curve in terms of frequency for ten modes in an elastic-viscoelastic three-layer adhesive joint. In this paper, the acceptable range of attenuation is calculated for ultrasonic inspection of the adhesive joint with $200 \mathrm{~mm}$ length using a single transducer. The suitable range of attenuation depends on wave propagation distance in a wave round-trip to the transducer and on the signal to noise ratio (SNR). In an inspection with guided waves, SNR is a measure for detecting small defects and is the ratio of reflected signal from defects to return signal from grains (as noise) to the transducer. A minimum identifiable SNR in guided wave test is $6 \mathrm{~dB}^{7}$ In a guided wave test, a defect signal is usually $20 \mathrm{~dB}$ higher than the noise signal; therefore, a $14 \mathrm{~dB}$ signal can be lost because of the guided wave mode attenuation, and if attenuation is more than $14 \mathrm{~dB}$, defects are not detectable. The distance that a wave travels in a round-trip to the transducer is twice the length of the plate and equal to $400 \mathrm{~mm}$, and maximum attenuation that the wave can have in a round-trip equals $-14 \mathrm{~dB} / 0.4 \mathrm{~m}$ or $-35 \mathrm{~dB} \mathrm{~m}^{-1}$, then the suitable range of attenuation is from 0 to $-35 \mathrm{~dB} \mathrm{~m}^{-1}$.

The modes with acceptable attenuation level (0 to $-35 \mathrm{~dB} \mathrm{~m}^{-1}$ ) are selected for inspection in high and low frequencies. In generating these modes, an adhesive joint inspection can be carried out to find the defects. From curves in Fig. 5, it can be seen that the $M 1$ mode in frequency range of $150 \mathrm{kHz}$ to $500 \mathrm{kHz}$ has a suitable attenuation level for inspection, and in frequencies higher than $500 \mathrm{kHz}$, a sudden and excessive increase can be seen in attenuation. This mode in high frequencies is not suitable for inspection. Attenuation in the $M 2$ mode in low frequencies, in the range of $150 \mathrm{kHz}$ to $250 \mathrm{kHz}$ increases extremely, and has a sudden and excessive increase in frequencies higher than $800 \mathrm{kHz}$. The $M 2$ mode in frequency range of $325 \mathrm{kHz}$ to $800 \mathrm{kHz}$ and the $M 3$ 


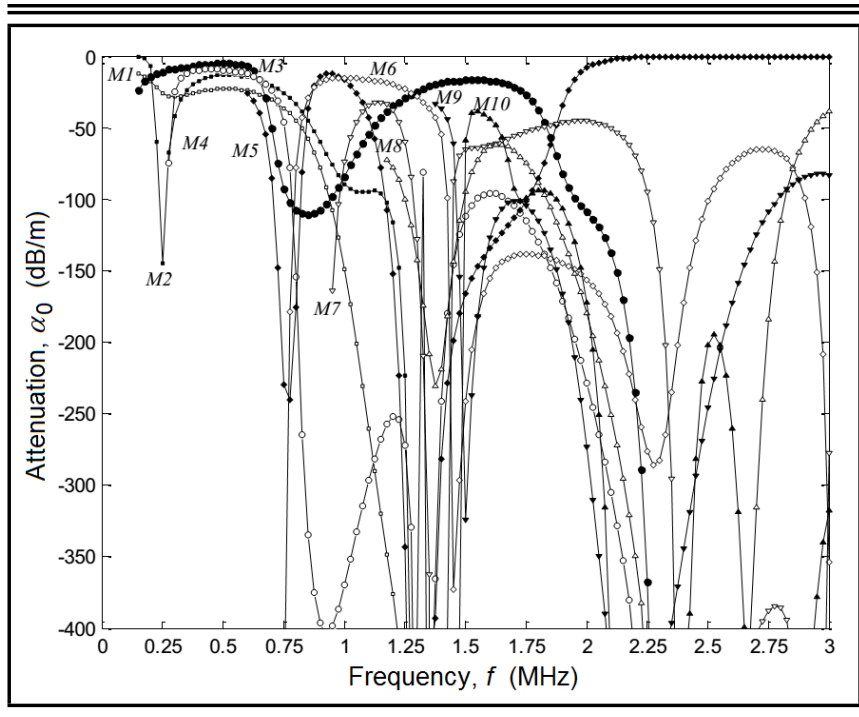

Figure 6. Attenuation dispersion curves in terms of frequency for 10 modes in the three-layer adhesive joint: aluminum-epoxy-aluminum.

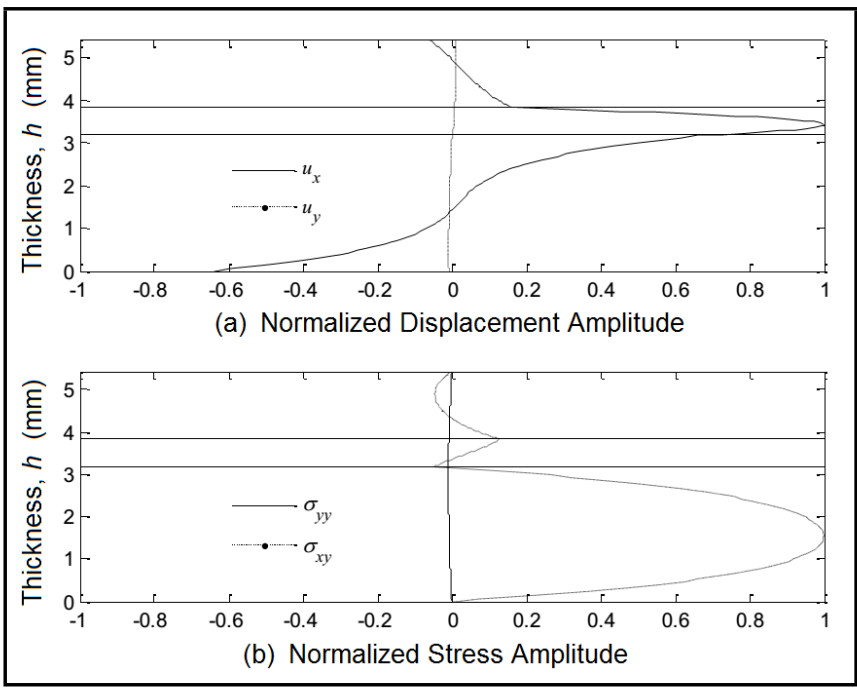

Figure 7. $M 2$ mode wave structure in $500 \mathrm{kHz}$ frequency with the attenuation of $-13.5 \mathrm{~dB} \mathrm{~m}^{-1}$ in a three-layer adhesive joint (a) normalized displacement wave structure and (b) normalized stress wave structure.

mode in frequency ranges of $150 \mathrm{kHz}$ to $675 \mathrm{kHz}$ and $1.2 \mathrm{MHz}$ to $1.775 \mathrm{MHz}$ have suitable attenuation levels for inspection of the adhesive joint with $200 \mathrm{~mm}$ length. The $M 3$ mode in $500 \mathrm{kHz}$ has an attenuation equal to $-5.1 \mathrm{~dB} \mathrm{~m}^{-1}$ which is the lowest attenuation level for this mode in frequency range of 0 to $3 \mathrm{MHz}$. The M5 mode has a suitable attenuation level in high frequencies and is suitable for inspection in $1.9 \mathrm{MHz}$ to $3 \mathrm{MHz}$ frequency range, and it also has negligible attenuation about $-0.27 \mathrm{~dB} \mathrm{~m}^{-1}$ in frequencies near $3 \mathrm{MHz}$.

\subsection{Validation of Numerical Solution Results}

One method to validate the numerical solution results of characteristic equations, which are the same as attenuation and phase velocity, is the investigation of the interfacial continuity equations and boundary conditions in the adhesive joint. The wave structure of the propagated modes in the three-layer adhesive joint is plotted to validate if the interfacial continuity equations and boundary conditions are satisfied. Wave structure curves are the same as stress and displacement amplitudes across three-layer adhesive joint thickness.

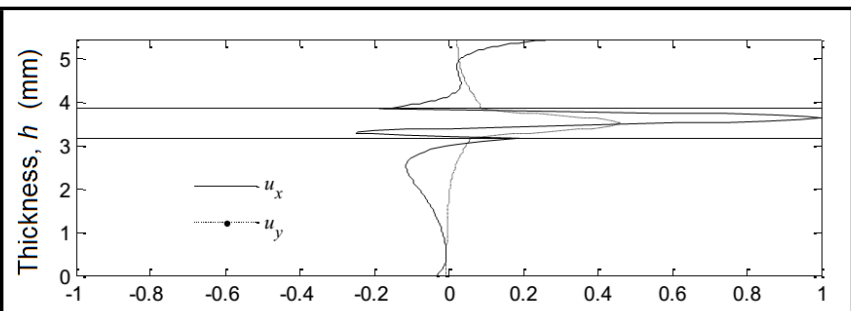

(a) Normalized Displacement Amplitude

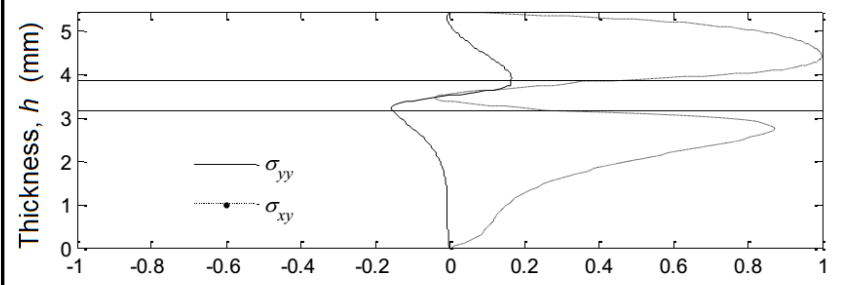

(b) Normalized Stress Amplitude

Figure 8. $M 2$ mode wave structure in $1.25 \mathrm{MHz}$ frequency with the attenuation of $-222.8 \mathrm{~dB} \mathrm{~m}^{-1}$ in a three-layer adhesive joint (a) normalized displacement wave structure and (b) normalized stress wave structure.

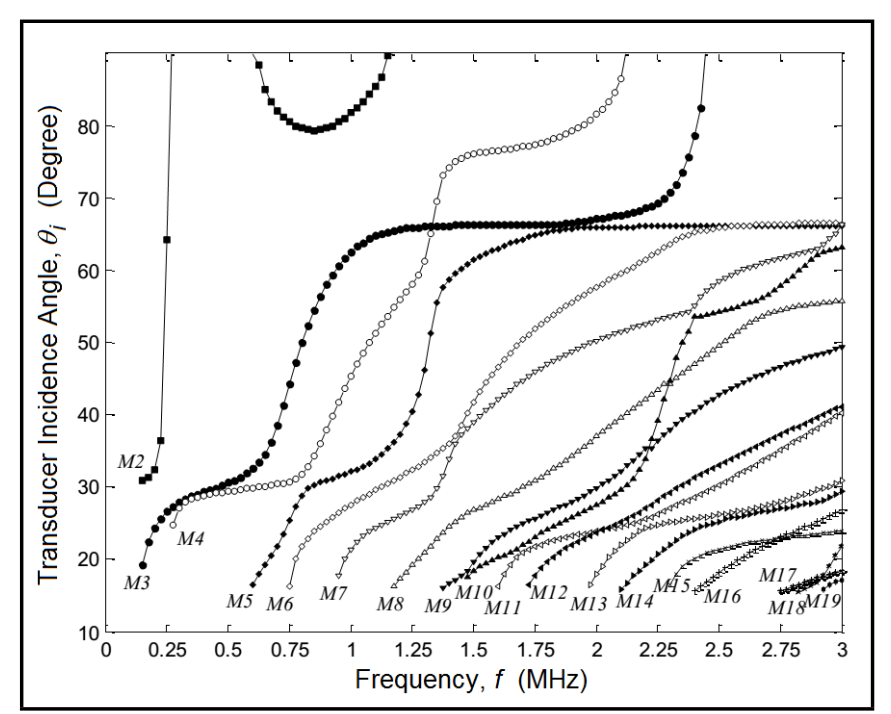

Figure 9. Transducer incidence angle curves in terms of frequency for generating Lamb wave modes in the three-layer adhesive joint: aluminum-epoxyaluminum.

Figure 7 shows the $M 2$ mode wave structure in $500 \mathrm{kHz}$ frequency with an attenuation of $13.5 \mathrm{~dB} \mathrm{~m}^{-1}$. As Fig. 7 shows, shear and normal stresses do not exist in free surfaces at the top and bottom of the three layers; also, interfacial continuity conditions including the continuity of shear and normal stresses and displacement components are satisfied. The $M 2$ mode wave structure in $1.25 \mathrm{MHz}$ frequency with the attenuation of $-222.8 \mathrm{~dB} \mathrm{~m}^{-1}$ is also plotted in the curves of Fig. 8, in which continuity and boundary conditions are also satisfied. Because attenuation level is high in wave structure curve of Fig. 8, most of the displacement exists in the viscoelastic layer.

\subsection{Transducer Incidence Angles to Generate Low-Attenuation Lamb Wave Modes}

In this section, at first the transducer incidence angle curves in terms of frequency for generating lamb wave modes in the three-layer adhesive joint is plotted using Eq. (31) and phase velocity values, these curves are shown in Fig. 9. Then the suit- 
able incidence angles are specified to generate low-attenuation modes by the transducer. In Fig. 9, it can be seen that the incidence angle for generating some modes such as the $M 1$ mode is 90 degree, the generation of which is impossible by the transducer. The $M 1$ mode cannot be generated by the transducer, because the transducer incidence angle for its generation is 90 degree for all the frequency ranges. The $M 2$ mode has a low attenuation level and a suitable incidence angle for inspection in $150 \mathrm{kHz}$ to $200 \mathrm{kHz}$ and $650 \mathrm{kHz}$ to $800 \mathrm{kHz}$ frequency ranges. To generate this mode in $200 \mathrm{kHz}$ frequency with low attenuation level of $-7.34 \mathrm{~dB} \mathrm{~m}^{-1}$, the transducer incidence angle should be 32.4 degree. Simultaneously investigating the attenuation values and the transducer incidence angle, it can be seen that the $M 3$ mode in $150 \mathrm{kHz}$ to $675 \mathrm{kHz}$ frequency range, the $M 4$ mode in $300 \mathrm{kHz}$ to $725 \mathrm{kHz}$ frequency range, and the $M 5$ mode in $875 \mathrm{kHz}$ to $1.075 \mathrm{MHz}$ and $1.9 \mathrm{MHz}$ to $3 \mathrm{MHz}$ frequency ranges have low attenuation level and suitable incidence angle for the generation of lamb wave. The transducer incidence angle should be 30.6 degree to generate the $M 3$ mode in $500 \mathrm{kHz}$ frequency and low attenuation level of $-5.1 \mathrm{~dB} \mathrm{~m}^{-1}$, and 66 degree to generate the $M 5$ mode in $3 \mathrm{MHz}$ frequency and negligible attenuation level of $-0.27 \mathrm{~dB} \mathrm{~m}^{-1}$.

\section{CONCLUSIONS}

The obtained results of lamb wave theoretical modeling in three-layer adhesive joint with a viscoelastic adhesive can be used in inspections using ultrasonic guided waves in threelayer structures. The results of the present paper can be summarized as:

1. Some modes, such as the $M 1, M 2$, and $M 3$, have an acceptable attenuation level for inspection with guided waves in low frequencies and some others, such as the M5 mode, in high frequencies.

2. Investigating the wave structure curves it can be seen that the interfacial continuity and boundary conditions is satisfied in adhesive joints. This result validates the numerical solution results of characteristic equation.

3. The transducer incidence angle obtained from theoretical modeling of lamb wave mode generation can be used to inspect adhesive joints and generate lamb wave with low attenuation level in joints.

4. To generate the $M 2$ mode in $200 \mathrm{kHz}$ frequency with low attenuation level of $-7.34 \mathrm{~dB} \mathrm{~m}^{-1}$, the transducer incidence angle should be 32.4 degree.

\section{REFERENCES}

1 Hosten, B. and Castaings, M. Transfer matrix of multilayered absorbing and anisotropic media: Measurements and simulations of ultrasonic wave propagation through composite materials, Journal of the Acoustical Society of America, 94, 1488-1495, (1993). http://dx.doi.org/10.1121/1.408152

2 Castaings, M. and Hosten, B. Delta operator technique to improve the Thomson-Haskell method stability for propagation in multilayered anisotropic absorbing plates, Journal of the Acoustical Society of America, 95, 1931-1941, (1994). http://dx.doi.org/10.1121/1.408707
3 Lowe, M. J. S. Matrix techniques for modeling ultrasonic waves in multilayered media, IEEE Transactions on Ultrasonics, Ferroelectrics, and Frequency Control, 42, 525542, (1995). http://dx.doi.org/10.1109/58.393096

4 Seifried, R., Jacobs, L. J., and Qu, J. Propagation of guided waves in adhesive bonded components, NDT \& E International, 35, 317-328, (2002). http://dx.doi.org/10.1016/s0963-8695(01)00056-1

5 Simonetti, F. Lamb wave propagation in elastic plate coated with viscoelastic materials, Journal of the Acoustical Society of America, 115, 2041-2053, (2004). http://dx.doi.org/10.1121/1.1695011

6 Simonetti, F. and Cawley, P. On the nature of shear horizontal wave propagation in elastic plates coated with viscoelastic materials, Proceedings of the Royal Society A, 460, 2197-2221, (2004). http://dx.doi.org/10.1098/rspa.2004.1284

7 Barshinger, J. N. and Rose, J. L. Guided wave propagation in an elastic hollow cylinder coated with a viscoelastic material, IEEE Transactions on Ultrasonics, Ferroelectrics, and Frequency Control, 51, 1547-1556, (2004). http://dx.doi.org/10.1109/tuffc.2004.1367496

8 Shorter, P. J. Wave propagation and damping in linear viscoelastic laminates, Journal of the Acoustical Society of America, 115, 1917-1925, (2004). http://dx.doi.org/10.1121/1.1689342

9 Birgersson, F., Finnveden, S., and Nilsson, C. M. A spectral super element for modelling of plate vibration-part 1: general theory, Journal of Sound and Vibration, 287, 297314, (2005). http://dx.doi.org/10.1016/j.jsv.2004.11.012

10 Bartoli, I., Marzani, A., Lanza di Scalea, F., and Viola, E. Modeling wave propagation in damped waveguides of arbitrary cross-section, Journal of Sound and Vibration, 295, 685-707, (2006). http://dx.doi.org/10.1016/j.jsv.2006.01.021

11 Puthillath, P. and Rose, J. L. Ultrasonic guided wave inspection of a titanium repair patch bonded to an aluminum aircraft skin, International Journal of Adhesion and Adhesives, 30, 566-573, (2010). http://dx.doi.org/10.1016/j.ijadhadh.2010.05.008

$12 \mathrm{Su}, \mathrm{Z}$. and $\mathrm{Ye}, \mathrm{L}$. Identification of damage using lamb waves: from fundamentals to applications, Springer-Verlag Press, Berlin Heidelberg, (2009). http://dx.doi.org/10.1007/978-1-84882-784-4

13 Rose, J. L. Ultrasonic waves in solid media, Cambridge University Press, Cambridge, (2004), paperback, 1st edition.

14 Christensen, R. M. Theory of viscoelasticity, Dover Publications, New York, (2010), paperback, 2nd edition.

15 Blanc, R. H. Transient wave propagation methods for determining the viscoelastic properties of solids, Journal of Applied Mechanics, 60, 763-768, (1993). http://dx.doi.org/10.1115/1.2900870 